CONFORMAL GEOMETRY AND DYNAMICS

An Electronic Journal of the American Mathematical Society

Volume 3, Pages 158-163 (December 2, 1999)

S $1088-4173(99) 00053-3$

\title{
EXAMPLES OF UNIFORMLY QUASIREGULAR MAPPINGS
}

\author{
KIRSI PELTONEN
}

\begin{abstract}
In this paper we construct examples of uniformly quasiregular (uqr) mappings. These provide counterexamples for a rigidity conjecture in quasiregular dynamics. It states that a closed manifold of dimension at least three, which admits a branched uqr mapping, is quasiconformally equivalent to an ordinary sphere $S^{n}$.
\end{abstract}

\section{Statements and RESUlts}

In this paper we construct examples of a certain type of quasiregular mappings. Topologically, quasiregular mappings are branched coverings, that is, covering mappings without the local injectivity requirement. This is a consequence of the completely analytic definition demanding that the mapping distorts the metric only by a bounded amount. More precisely:

Definition 1. A continuous mapping $f: G \rightarrow \mathbf{R}^{n}$ of a domain $G \subset \mathbf{R}^{n}$ is quasiregular, if it

$$
\text { belongs to } W_{n, l o c}^{1}(G)
$$

and if there exists a number $K, 1 \leq K<\infty$, such that

$$
\left|f^{\prime}(x)\right|^{n} \leq K J_{f}(x)
$$

holds almost everywhere in $G$.

In the above definition $W_{n, l o c}^{1}(G)$ is the Sobolev space of mappings with weak first order partial derivatives which are locally $L^{n}$ integrable. For the above mappings the partial derivatives in the ordinary sense exist almost everywhere. We can thus define the formal derivative of $f$ in terms of partial derivatives. The Jacobian determinant $\operatorname{det} f^{\prime}(x)$ of $f$ at $x$ is denoted by $J_{f}(x)$. By the norm of the linear mapping $f^{\prime}(x)$ we mean here the operator norm

$$
\left|f^{\prime}(x)\right|=\sup _{|h|=1}\left|f^{\prime}(x) h\right| .
$$

The above definition generalizes immediately to Riemannian manifolds. The branch set $B_{f}$ is the set of points for which $f$ is not locally homeomorphic. For the basic properties and theory of quasiregular mappings see $[\mathrm{R}]$.

Received by the editors July 26, 1999 and, in revised form, October 27, 1999.

1991 Mathematics Subject Classification. Primary 30C65, 58F; Secondary 53C.

Key words and phrases. Uniformly quasiregular mappings, quasiregular dynamics, branched covering.

(C)1999 American Mathematical Society 
Definition 2. A mapping $g: M^{n} \rightarrow M^{n}$ is uniformly quasiregular if all its $m$-fold iterates $g^{m}$ (compositions with itself) are quasiregular with a uniform bound on the dilatation.

For the motivation of this terminology coming from groups of quasiconformal mappings and basic properties see $[\mathrm{IM}]$ and $[\mathrm{M}]$. The theory of quasiregular dynamics is further developed in $\mathrm{MM}$.

Theorem 3. Let $M^{n}$ be a spherical space form. Then $M^{n}$ admits a branched uniformly quasiregular mapping.

A spherical space form $M^{n}$ is a smooth complete, connected Riemannian manifold of constant sectional curvature $K>0$. These can be characterized as spaces isometric to quotients $S^{n} / \Gamma$, where $S^{n}=S^{n}\left(\frac{1}{\sqrt{K}}\right) \subset \mathbf{R}^{n+1}$ is equipped with the standard metric and $\Gamma \subset O(n+1)$ acts freely and properly discontinuously [W]. If we consider $M^{n}$ equipped with this metric giving positive constant curvature, the corresponding covering map $h: S^{n} \rightarrow M^{n}$ is an isometry whenever an injection. Any smooth manifold $M^{n}$ with $S^{n}$ as a universal covering space and fundamental group $\Gamma$ acting properly discontinuously on $S^{n}$ gives a spherical space form isometric to $S^{n} / \Gamma$. Complete classification of spherical space forms is given in [W].

It turns out that the metric is not an obstruction here and the situation is even more general. Namely we have the following.

Theorem 4. Let $M^{n}$ be a smooth Riemannian manifold with universal covering space $S^{n}$. Then $M^{n}$ admits a branched uniformly quasiregular mapping.

Theorem 5. Let $M^{n}$ be as in Theorem 4. Then any quasiregular mapping $f: M^{n} \rightarrow S^{n}$ composed by the covering mapping $S^{n} \rightarrow M^{n}$ can be deformed to a uniformly quasiregular mapping $M^{n} \rightarrow M^{n}$.

In the above theorem by a deformation of a quasiregular mapping $M^{n} \rightarrow M^{n}$ we mean a uniformly quasiregular mapping $M^{n} \rightarrow M^{n}$ with the same branch set.

Theorem 6. Theorems 4 and 5 hold if in place of a covering mapping there is a branched covering mapping.

Corollary 7. The Poincaré homology sphere and the Lens spaces in dimension 3 admit uniformly quasiregular mappings.

The proof of the above statements is by construction and essentially a modification of a trick invented by G. Martin and T. Iwaniec [IM], [M]. We construct an analogous conformal trap, where a ball is conformally mapped into itself. The two essential steps in building the trap in [M] are the following:

(1) A local modification of the original quasiregular mapping $S^{n} \rightarrow S^{n}$ to become a translation of the trap outside itself.

(2) Inversion with respect to the boundary of the trap.

Step one generalizes immediately to a quasiregular map $M^{n} \rightarrow S^{n}$. The only global property needed in the construction is the inversion (2). This step we can take in a suitably chosen ball which is mapped further onto the trap by the covering mapping.

The dynamics of these mappings are the same as for maps in $[\underline{M}$. See also $[\mathrm{HM}]$. 


\section{Proofs}

Proof of Theorem [3. Assume $M$ is a spherical space form of constant sectional curvature $K>0$. Denote by $S^{n}=S^{n}\left(\frac{1}{\sqrt{K}}\right) \subset \mathbf{R}^{n+1}$ the sphere of radius $\frac{1}{\sqrt{K}}$ centered at the origin of $\mathbf{R}^{n+1}$ with the standard metric. We equip $M^{n}$ with the natural metric for which $h: S^{n} \rightarrow M^{n}=S^{n} / \Gamma$ is a local isometry. Then we take an Alexander type mapping $f: M^{n} \rightarrow S^{n}$, which is constructed as follows. Divide the compact manifold into finitely many simplices and further take a barycentric subdivision by adding a new vertex into each of the faces. In this situation there is always an even number of simplices attached to each of the $(n-2)$-faces. Consider then the sphere $S^{n}$ divided into two by upper and lower hemispheres. The simplices of the manifold $M^{n}$ are then mapped pairwise to the two pieces of the sphere respectively. The branch set of the mapping is exactly the $(n-2)$-skeleton. Assume that the degrees of the mappings $f$ and $h$ are $p$ and $q$ respectively. The construction works as well for any other quasiregular mapping available from $M^{n}$ onto $S^{n}$ in place of $f$.

Let $x_{0} \in M^{n}$ be any point outside the branch set. Denote by $y_{0}$ one of the points in the set $h^{-1}\left(x_{0}\right)$. One can assume that $y_{0} \neq f\left(x_{0}\right)$. We will build a conformal trap to the neighbourhood of the point $x_{0}$ by making an inversion in the neighbourhood of the point $y_{0}$ analogously as in the construction in $[\mathrm{M}]$. Analogously we need the following properties:

Set $f^{-1}\left(y_{0}\right)$ and $x_{0}$ do not meet $B_{f}$.

There are small, disjoint balls $U_{0}=B\left(f\left(x_{0}\right), r\right)$ and $V_{0}=B\left(y_{0}, r\right)$ about $f\left(x_{0}\right)$ and $y_{0}$ in $S^{n}$ such that $f^{-1}\left(U_{0}\right)$ has a component $U_{1}$ containing $x_{0}$ and $f^{-1}\left(V_{0}\right)$ has components $V_{1}, \ldots, V_{p}$ pairwise disjoint and such that $\left.f\right|_{U_{1}}: U_{1} \rightarrow U_{0},\left.f\right|_{V_{i}}: V_{i} \rightarrow V_{0}$ and $\left.h\right|_{V_{0}}: V_{0} \rightarrow h\left(V_{0}\right)$ are injective.

The above can be organized for almost all pairs of points $\left(x_{0}, y_{0}\right)[\underline{\mathrm{R}}$. Denote by $\left\{y_{1}, \ldots, y_{p}\right\}=f^{-1}\left(y_{0}\right)$. Let $a, b>0$ be so small that $2 b<a$ and the following conditions are satisfied:

$$
\begin{aligned}
& B\left(x_{0}, a\right) \subset U_{1}, B\left(y_{i}, a\right) \subset V_{i}, i=1, \ldots, p, \\
& B\left(y_{0}, b\right) \subset \bigcap_{i=1}^{p} f\left(B\left(y_{i}, a\right)\right), B\left(f\left(x_{0}\right), b\right) \subset f\left(B\left(x_{0}, a\right)\right), \\
& f\left(B\left(x_{0}, b\right)\right) \subset B\left(f\left(x_{0}\right), a\right), f\left(B\left(y_{i}, b\right)\right) \subset B\left(y_{0}, a\right), i=1, \ldots, p .
\end{aligned}
$$

Now define an intermediary map $g_{1}$ as follows. On

$$
M^{n} \backslash\left\{\bigcup_{i=1}^{p} B\left(y_{i}, a\right) \cup B\left(x_{0}, a\right)\right\}
$$

we set $g_{1}=f$. For $1 \leq i \leq p$ we map $B\left(x_{0}, b\right)$ onto $B\left(f\left(x_{0}\right), b\right)$ and $B\left(y_{i}, b\right)$ onto $B\left(y_{0}, b\right)$ isometrically. On the annular regions $\left.g_{1}\right|_{B\left(x_{0}, a\right) \backslash B\left(x_{0}, b\right)}$ and $\left.g_{1}\right|_{B\left(y_{i}, a\right) \backslash B\left(y_{i}, b\right)}$ is obtained for each $i=1, \ldots, p$ by application of Sullivan's version of the Annulus Theorem for quasiconformal mappings TV]. In the above $\left.g_{1}\right|_{\partial B\left(x_{0}, a\right)}=f$, $\left.g_{1}\right|_{\partial B\left(y_{i}, a\right)}=f$ and $\left.g_{1}\right|_{\partial B\left(x_{0}, b\right)},\left.g_{1}\right|_{\partial B\left(y_{i}, b\right)}$ is the appropriate isometry. The conditions (3) -(5) imply the map $g_{1}: M^{n} \rightarrow S^{n}$ is well defined and quasiregular. Additionally we have by construction $B_{g_{1}}=B_{f}$. 
Next let $\Phi: S^{n} \rightarrow S^{n}$ be a conformal mapping which exchanges $B\left(y_{0}, b\right)$ with its complement and set

$$
g=h \circ \Phi \circ g_{1}: M^{n} \rightarrow M^{n}
$$

Clearly $B_{g}=B_{f}$. Next we show that $g$ and all its iterates are uniformly quasiregular. Denote by $B=B\left(x_{0}, b\right)$ the conformal trap. Restriction map $\left.g\right|_{B}$ is conformal and

$$
g(\bar{B})=h\left(\overline{\Phi\left(B\left(f\left(x_{0}\right), b\right)\right)}\right) \subset h\left(B\left(y_{0}, b\right)\right)=B\left(h\left(y_{0}\right), b\right)=B
$$

by (5) and conformality of $h$. It follows that $\left.g^{m}\right|_{B}=\left.g \circ \cdots \circ g\right|_{B}$ is conformal for all $m \geq 1$. Assume next that $x \in M^{n} \backslash\left\{\bigcup_{i=1}^{p} B\left(y_{i}, b\right) \cup B\left(x_{0}, b\right)\right\}$. Then $g_{1}(x) \in$ $S^{n} \backslash B\left(y_{0}, b\right)$ and hence $\Phi\left(g_{1}(x)\right) \in \bar{B}\left(y_{0}, b\right)$ and finally $g(x) \in \bar{B}$ since $h$ is an isometry in $B\left(y_{0}, b\right)$. It follows that $\left.g^{m}\right|_{M^{n} \backslash\left\{\bigcup_{i=1}^{p} B\left(y_{i}, b\right) \cup B\left(x_{0}, b\right)\right\}}$ is quasiregular for each $m$ with a uniform bound on the dilatation by our first observation. Finally if $x \in B\left(y_{i}, b\right), i=1, \ldots, p$, then $g$ is a conformal isometry followed by a conformal $\Phi$ and $h$ as above. Thus the iterates stay conformal at $x$ until it passes into the complement of $\bigcup_{i=1}^{p} B\left(y_{i}, b\right) \cup B\left(x_{0}, b\right)$. Then it picks up some distortion before passing into $B$ and the iterates again stay conformal.

Proof of Theorem 4. If the manifold $M^{n}$ is equipped with an arbitrary Riemannian metric, it would be tempting to take the induced metric by the covering mapping on the sphere, making the covering $h: S^{n} \rightarrow M^{n}$ conformal. However, in this situation the inversion $\Phi$ need not be conformal. This was pointed out by V. Mayer. We equip the sphere with the standard spherical metric and modify the covering mapping, which is locally quasiconformal, as we modified the mapping $f$ in the proof of Theorem 3 . We proceed exactly as in the proof of Theorem 3 and use the same notation. The modification for $h$ needs to be done in small neighbourhoods of $y_{0}$ and $h^{-1}\left(y_{i}\right), i=1, \ldots, p$. We do the following additions. Denote $\left\{z_{i}^{1}, \ldots, z_{i}^{q}\right\}=h^{-1}\left(y_{i}\right), i=1, \ldots, p$. We choose $r>0$ here so small that in addition to the conditions in the proof of Theorem 3 , also sets $\left\{W_{i}^{1}, \ldots, W_{i}^{q}\right\}=h^{-1} B\left(y_{i}, r\right), i=1, \ldots, p$, and $V_{0}$ are pairwise disjoint and $\left.h\right|_{W_{i}^{j}}: W_{i}^{j} \rightarrow h\left(W_{i}^{j}\right),\left.h\right|_{V_{0}}: V_{0} \rightarrow h\left(V_{0}\right)$ are injective for all $i=1, \ldots, p$, $j=1, \ldots, q$. Further we let $a, b>0$ be so small that the following conditions are also satisfied:

$$
\begin{aligned}
& B\left(z_{i}^{j}, a\right) \subset W_{i}^{j}, j=1, \ldots, q, i=1, \ldots, p \\
& B\left(y_{i}, b\right) \subset \bigcap_{i=1}^{q} h\left(B\left(z_{i}^{j}, a\right)\right), i=1, \ldots, p, B\left(x_{0}, b\right) \subset h\left(B\left(y_{0}, a\right)\right), \\
& h\left(B\left(z_{i}^{j}, b\right)\right) \subset B\left(y_{i}, a\right), j=1, \ldots, q, i=1, \ldots, p \\
& h\left(B\left(y_{0}, b\right)\right) \subset B\left(x_{0}, a\right) .
\end{aligned}
$$

We define an intermediary map $h_{1}$ on $S^{n} \backslash\left\{\bigcup_{i, j=1}^{p, q} B\left(z_{i}^{j}, a\right) \cup B\left(y_{0}, a\right)\right\}$ by setting $h_{1}=h$. For $j=1, \ldots, q$ we map $B\left(z_{i}^{j}, b\right)$ onto $B\left(y_{i}, b\right)$ and $B\left(y_{0}, b\right)$ onto $B\left(x_{0}, b\right)$ isometrically for each $i=1, \ldots, p$ and extend to annular regions as in Theorem 3 . We claim now that the mapping $g=h_{1} \circ \Phi \circ g_{1}$ is uqr. The conformal trap and 
points in $M^{n} \backslash\left\{\bigcup_{i=1}^{p} B\left(y_{i}, b\right) \cup B\left(x_{0}, b\right)\right\}$ behave as in the proof of Theorem 3. If

$$
x \in g_{1}^{-1}\left(\Phi^{-1}\left(h_{1}^{-1}\left(B\left(y_{i}, b\right)\right)\right)\right) \subset \bigcup_{i=1}^{p} B\left(y_{i}, b\right),
$$

then $g(x)$ ends up to the set $\bigcup_{i=1}^{p} B\left(y_{i}, b\right)$ under conformal steps. If

$$
x \in B\left(y_{i}, b\right) \backslash g_{1}^{-1}\left(\Phi^{-1}\left(h_{1}^{-1}\left(B\left(y_{i}, b\right)\right)\right)\right),
$$

then

$$
\Phi\left(g_{1}(x)\right) \in S^{n} \backslash\left\{B\left(y_{0}, b\right) \bigcup_{i=1}^{p} h_{1}^{-1}\left(B\left(y_{i}, b\right)\right)\right\}
$$

and $g(x)$ ends up to $M^{n} \backslash \bigcup_{i=1}^{p} B\left(y_{i}, b\right)$ picking up some dilatation under $h_{1}$. After that $g_{1}$ can pick up some dilatation again but then $g_{1}(g(x)) \in S^{n} \backslash B\left(y_{0}, b\right)$ and $g^{2}(x) \in \bar{B}\left(x_{0}, b\right)$ ending the trap.

Proof of Theorem [5. The statement follows immediately since our construction does not make any explicit use of the Alexander mapping $M^{n} \rightarrow S^{n}$.

Proof of Theorem [6. The similar construction for the branched covering mapping $h: S^{n} \rightarrow M^{n}$ is possible by choosing the relevant points outside the branch set of the corresponding mappings. It is enough to choose $x_{0}$ outside the set $B_{f} \cup h B_{h} \cup h f h B_{h}$ and such that $f\left(x_{0}\right)$ is outside $B_{h}$. The set of such points is dense in $M^{n}$. In this situation none of the points $y_{0}, h^{-1}\left(y_{i}\right), i=1, \ldots, p$, belongs to $B_{h}$ and $h$ can be modified to be an isometry in small neighbourhoods of those points.

Proof of Corollary 7 The statement is evident. The Poincaré homology sphere and Lens spaces are interesting 3-dimensional examples of manifolds whose universal covering space is the 3-sphere. The Poincaré homology sphere has fundamental group of order 120. For its several descriptions see [KS] and a detailed dodecahedral construction see $\underline{\mathrm{P}}$. It is an example of a homogeneous spherical space form. Lens space $L(p, q)=S^{3} /(\mathbf{Z} / p)$ has fundamental group of order $p$. For these and more examples in dimension 3 see [Mo and also in higher dimensions see [W].

\section{ACKNOWLEDGEMENTS}

This paper was born after an inspiring lecture on open questions in the field of uqr mappings by Gaven Martin in Haifa, May 1999, and patient explanations by Volker Mayer on some related constructions. The author thanks both gentlemen for dynamic discussions.

\section{REFERENCES}

[HM] A. Hinkkanen and G.J. Martin, Attractors in quasiregular semigroups. Proc. XVI Nevanlinna colloquim, Eds. I. Laine and O. Martio, de Gruyter, Berlin-New York, 1996, 135-141. MR 97m:30028

[IM] T. Iwaniec and G.J. Martin, Quasiregular semigroups, Ann. Acad. Sci. Fenn. 21, 1996, 241-254. MR 97i:30032

[KS] R. Kirby and M. Scharlemann, Eight faces of Poincaré homology 3-sphere, Proc. of Geometric Topology Conference, Athens, 1979. MR 80k:57042

[M] G.J. Martin, Branch sets of uniformly quasiregular maps, Conformal Geometry and Dynamics 1, 24-27. MR 98d:30032

[MM] G.J. Martin and V. Mayer, Rigidity in holomorphic and quasiregular dynamics. Preprint.

[Mo] J.M. Montesinos, Classical tessellations and three-manifolds, Springer-Verlag, 1987. MR 89d:57016 
[P] K. Peltonen, Quasiregularity on homology manifolds, MLI preprint no 14, 95/96.

[R] S. Rickman, Quasiregular mappings, Springer-Verlag, 1993. MR 95g:30026

[TV] P. Tukia and J. Väisälä, Lipschitz and quasiconformal approximation and extension, Ann. Acad. Sci. Fenn. Ser. A I Math. 6, 1981, 303-342. MR 84a:57016

[W] J.A. Wolf, Spaces of constant curvature, McGraw-Hill, 1967. MR 36:829

Helsinki University of Technology, Department of Engineering Physics and Mathematics, P.O. Box 1100, 02015 HUT, Finland

E-mail address: Kirsi.Peltonen@hut.fi 